\title{
IMAGE PROCESSING TECHNIQUES FOR FAST AND ACCURATE ESTIMATION OF POSE OF A DOUBLE PENDULUM
}

\author{
Muhammad Haris, Junaid Ahmed Memon and Muhammad Farhan \\ School of Science and Engineering, Habib University, Karachi, 75290 Sindh, Pakistan
}

\begin{abstract}
Double pendulum exhibits a rich dynamic behavior and is often used to study nonlinear dynamics, instability, and chaos. In order to devise a method for its control, the angles and the angular velocities of its two arms need to be measured accurately with sufficiently fast sampling rate. Generally, encoders and other sensors are used which are costly, difficult to install for custom built pendulums, and require continuous calibration to measure the angles. Therefore, in this paper we present a comparison of four image processing based methods to estimate the angles of double pendulum. The first two methods are widely used involving variants of Canny edge detection in conjunction with Hough transform. The third method extracts the skeleton of the thresholded pendulum and then applies Hough transform on the skeleton to compute the angles. The fourth method, never before used for double pendulum, segments the pendulum arms and uses basic matrix operations to extract the angles. The results focus on the speed-accuracy-memory tradeoff for all the methods and manifest that the fourth method is satisfactorily accurate with minimum computational complexity and memory usage.
\end{abstract}

\section{KEYWORDS}

Double Pendulum, Pose Estimation, Canny Edge Detection, Hough Transform, Speed-Accuracy Tradeoff

\section{INTRODUCTION}

Accurate position control is a problem in robotic systems. In many applications, robot bodies are assumed to be rigid, however, it is not true for all cases. Therefore, the analysis and control of the physical model of a flexible robot and solving its tracking problem is a useful topic in robotics and has been considered in many literature (Artega, 2000), (Lee, 1998). Double pendulum is one such system, where two arms are linked with non-rigid joint. The study of double pendulum is important in the field of physics and especially in control engineering (Ataei, 2007) where the general interest lies in its control. In order to control the system it is necessary to track pendulum arms by knowing the angles of the double pendulum. There are a few ways to track pendulum arm e.g., with Inertial Measurement Unit using sensors (accelerometer and gyroscopes), using encoders and via image processing (Kiril, 2013). Error accumulation (Kiril, 2013) and noise, and requirement to modify setup are major problems when sensors are used whereas use of image processing algorithm enables a rather accurate tracking at a lower cost with ease in installation.

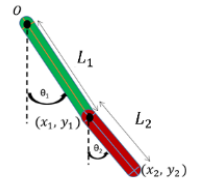

Figure 1. Diagram demonstrating the project

In order to control the pendulum, the angles the arms make with the vertical axis need to be fed into the mathematical model to produce appropriate output voltage for the motors. Our focus is to extract the pose of double pendulum i.e. $\theta_{1}$ and $\theta_{2}$ as shown in Figure 1. There can be a variety of algorithms to move forward with the angle estimation from a series of images of a pendulum. One method is by marking the joints physically (Kulp, 2007) where background color and light intensity plays an important role and extracting coordinates of joints. After discriminating the joints, the displacement, $\left(x_{i}, y_{i}\right)$, from the reference origin can be calculated. This displacement can be converted to angles, $\theta_{i}$ using the following mathematical formula (Kulp, 2007). 


$$
\theta_{i}=\operatorname{atan} 2\left(y_{i}-y_{i-1}, x_{i}-x_{i-1}\right)
$$

There can be a number of ways to segment out joints. For the ease of the process, markers be physically placed on joints so that bounding boxes can be obtained easily. Other approaches include detecting edges of the pendulum arms and segmenting it. There are quite a few approaches within this domain as well. For example, the authors in (Ali, 2015) proposed to use Hough transform on lines, corresponding to arms, extracted from the image to measure angles of the double pendulum by applying appropriate trigonometry, and their method reported maximum error of $0.4^{\circ}$. It is to go from the $(x, y)$ space to the $(\rho, \phi)$ space in order to detect simple shapes like line, circle or ellipse. Figure 2 demonstrates the mapping.

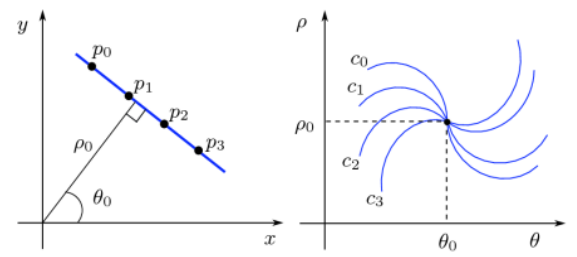

Figure 2. Change of space during Hough transform (Bergues, 2019)

We can see that where there are intersection in the $(\rho, \phi)$ space, they correspond to a line in the $(x, y)$ space. The same paper proposes other ways which include detecting pointers or markers corresponding to end-points as well as defining points in space and using pattern recognition to find the angles. However, the paper concludes that use of Hough transform yields the most accurate angles.

Another paper (Kavithaa, 2013) used vision algorithm called Lukas Kanade's Optical Flow to track the blob. The histogram equalization based pre-processing is applied and thresholding is performed to detect the blob. They used a blob library in OpenCV to detect the blob. Repeated edge detection and Hough transform is applied on each image to get the track in another paper (Ngyuyen, 2009).

Another paper (Robinson, 2010), cited that it was very difficult to extract actual angular measurements from the radial and tangential accelerations provided by the accelerometer and that the resolution provided was insufficient to yield clear data, even after filtering. Therefore, they shifted to vision-based approach to track the double pendulum arms. They extracted 8-bit image frame of the captured video. The 8-bit image is then morphologically opened to remove noise in the binary image. Following opening, thinning is applied to the image to extract the "skeleton" of the image and the endpoints are found which are used to get the angles.

From literature, it was found that Canny edge detection followed by Hough transform is a reliable way to get angles, however these algorithms are slow. We present a comparison of four methods in terms of speed, accuracy and memory consumption so that one can choose a method based on the performance requirement.

\section{METHODOLOGY}

In this paper we compare four methods to estimate the pose of a double pendulum. The machine used to run the algorithms had a 64-bit $2.4 \mathrm{GHz}$ processor with 4 GB RAM. The camera captures video at a resolution of $1080 \mathrm{p}$ at a frame rate of $30 \mathrm{fps}$. Before applying any of these methods, pre-processing is required to remove noise and sharp edges in the background occurring mainly due to uneven lighting. Blurring using average filter is applied on the image after it is converted to grayscale from RGB.

In order to evaluate performance we run each of the following algorithms on 300 frames. The metrics used to compare these methods include memory consumption, time taken to compute angles for each frame and RMS error in angles from the true values. These true values are obtained by segmenting out small markers from the pendulum. For the benchmark data we use images of pendulum with markers on it. Using basic thresholding, $T_{1}>P(x, y)>T_{2}$, the markers placed on arms are segmented out. The markers are labelled and three points on pendulum are extracted out and the angles are computed using the formula given in equation (1).

\subsection{Canny Edge Detection Followed by Hough Transform (Method 1)}

This method uses Canny edge (Canny, 1986) detection to extract single pixel thick edges of the double pendulum. Hough transform (Richard, 1972) is then applied on these edges and the three peaks with the highest values, in the Hough space, are used which gives the angles of these lines. These three peaks 
correspond to the two lines for the upper arm and one for the lower arm. The first two peaks have the same angle. The two unique angles are chosen, directly from the Hough space. Figure 3 shows a frame being processed using the described method.
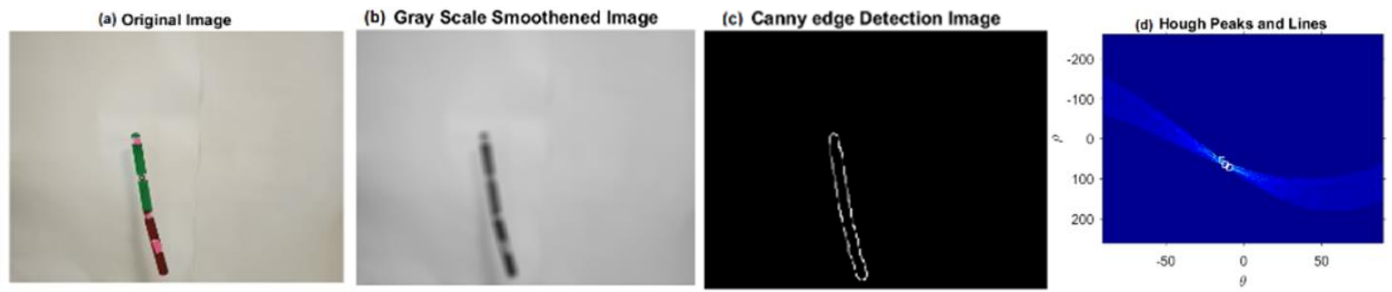

Figure 3. Processing via method 1. (a) Original Image. (b) Gray Scale Image. (c) Canny Edge Image. (d) Hough Peaks

\subsection{Optimized Canny Edge Detection Followed by Hough Transform (Method 2)}

Approximate Canny Edge (Miroslav, 2018) is builtin function in MATLAB and it makes the entire procedure nearly 2.7 times faster than the above method. The optimal function in Canny's detector is described by the sum of four exponential terms, but it can be approximated by the first derivative of a Gaussian. This method is exactly like method 2. Approximate Canny Edge is used to detect edges of the segmented pendulum. Once the edge is found, the method uses Hough transform for detecting angles as was in the previous case, by connecting lines in the edge image. This yields lines and their corresponding angles. The first and the third peaks' angles are selected since they are unique and correspond to the upper and lower arm respectively.

\subsection{Hough Transform of Skeleton Image (Method 3)}

This method computes the skeleton, by repeated application of morphological operations, namely erosion and dilation, on the pendulum image followed by applying Hough transform on it. In this method only the two highest valued peaks are utilized. The two peaks taken into consideration correspond to the upper and the lower arm respectively. Once again, the angles are extracted directly from the Hough space. Figure 4 (a), (b) shows a frame being processed using the described method. Hough transform detects two lines (pendulum arms) in the $(\rho, \phi)$ space, which directly yields the angle.

\subsection{Constraints based Image thresholding (Method 4)}

In this method we exploit the white background and threshold the image to separate foreground from the background. This method requires some information about the fixed joint of the pendulum, $O$, and the length of both the arms, $L_{1}$ and $L_{2}$. The algorithm searches for the closest high pixel in the vicinity of $O$, then looks for a high pixel at a distance $L_{1}$ from the first point. The last step is to detect a high pixel at a distance $L_{2}$ from the second point. Algorithm makes sure that the third point does not end up on the first arm rather it is chosen depending on the angle $\theta_{1}$. Each search returns an array of pixels, and for whilst searching for the third point either the first index or the last index is chosen, depending on whether the first arm makes a negative or a positive angle with the vertical axis, respectively. The operations are simple which greatly increases its speed, unlike other methods where morphological and complex algorithms are being used. Figure 4 (b), (c) shows a frame being processed using the described method. However, this method places some constraints on the length of the pendulum, hence if the working distance is altered the length needs to be calibrated manually.
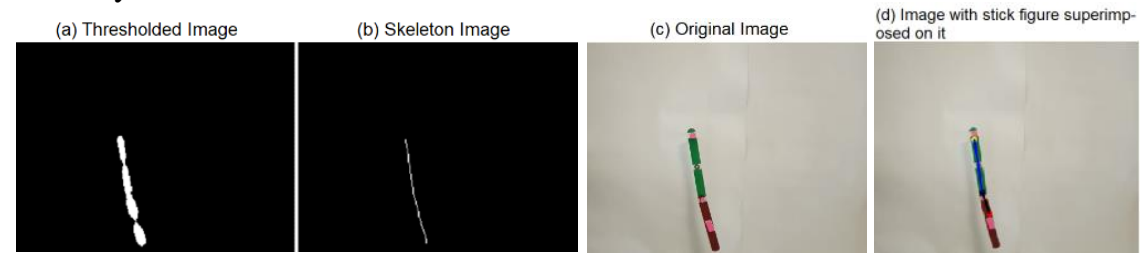

Figure 4. Frame being processed using method 3, 4. (a) Thresholded Image. (b) Skeleton Image (c) Original Image.

(d) Image with stick figure superimposed on it 


\section{RESULTS AND DISCUSSION}

Figures 5 and 6 compare the error in angles, $\theta_{1}$ and $\theta_{2}$, the computation time, and memory usage for the four methods with respect to changing image scale. The first 3 methods are satisfactorily fast, whereas the last method is the fastest. Method 3 improves the speed of computation by optimizing the edge detection procedure. The last method yields the least error for the first limb, however, for the second limb the last method yields the highest error. The error for second limb stays the same for the first three methods, so they are consistent. The memory consumption is the lowest for the last method, and nearly the same for the first three methods.
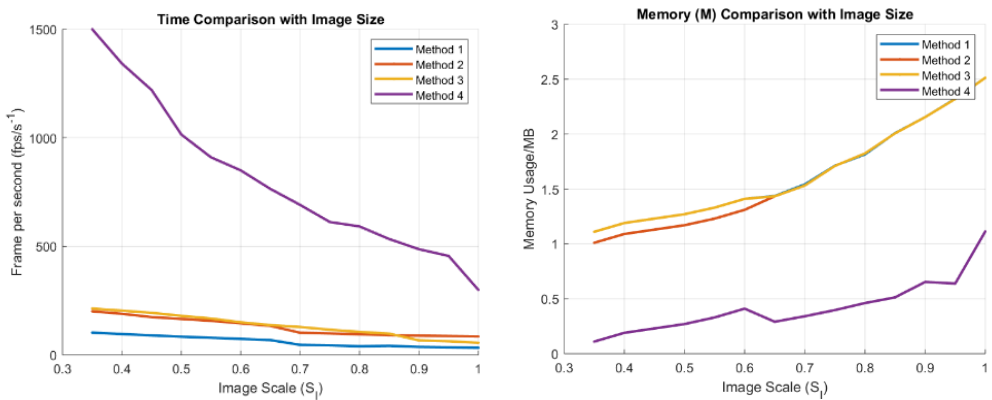

Figure 5. Speed and Memory for different methods. (a) Time Comparison for different Methods from reference data Frames processed per second fps against Image Scale (left). (b) Memory Usage Comparison for different Methods from reference data (MBs used against Image Scale) (right)
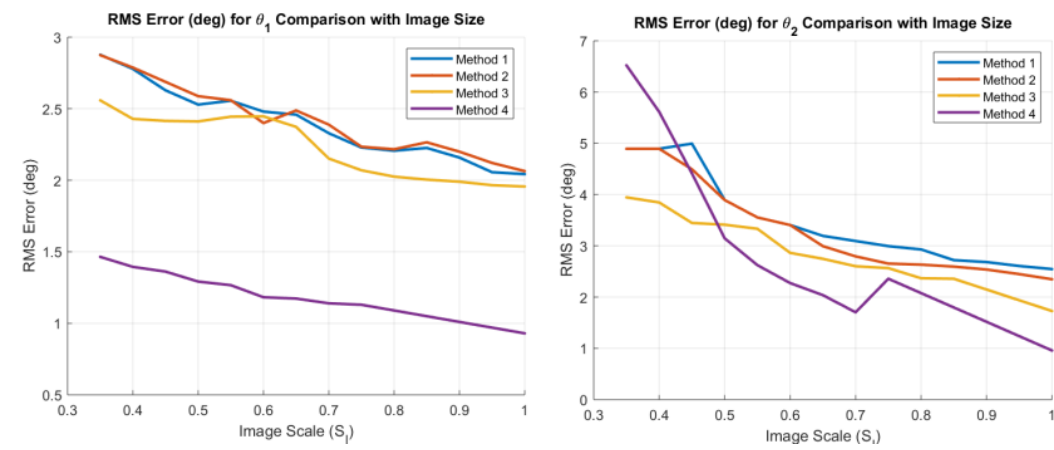

Figure 6. Accuracy Comparison for different methods from reference data. (a) RMS error (deg) for limb-1 against Image Scale) (left). (b) RMS error (deg) for limb-2 against Image Scale) (right)
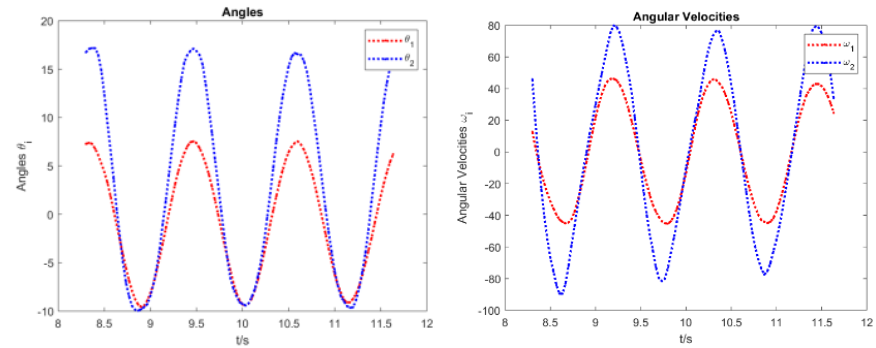

Figure 7. Results obtained (a) Angles obtained (Left), (b) Angular velocities (Right)

For the graph comparing time in Figure 5(a), the first three methods demonstrate a nearly constant behavior, however, a quadratic function best fits the data for the fourth method. The method used to derive the relation is obtained using least squares method. Here $S_{I}$ is the image scale. For the graph comparing memory consumption, 5(b), a similar trend is seen; the first three methods fit a single quadratic function and the fourth method fits a separate linear function.

In the graphs comparing the RMS error in $\theta_{1}$, in Figure 6(a), we can see that a single linear functions fits the first three methods and a separate linear function fits the data for the fourth method. In the graphs 
comparing RMS errors in $\theta_{2}$, in Figure 6(b), we can see that all the curves can be approximated by a single quadratic function, with a reasonable degree of error.

The following table shows standard deviation in error in angles for different methods.

Table 1. Standard deviation in error in angles for different methods

\begin{tabular}{|c|c|c|c|c|}
\hline $\boldsymbol{-}$ & Method 1 & Method 2 & Method 3 & Method 4 \\
\hline $\boldsymbol{\theta}_{\mathbf{1}}$ & 3.5361 & 3.3476 & 8.4567 & 0.4176 \\
\hline $\boldsymbol{\theta}_{\mathbf{2}}$ & 2.9545 & 3.2314 & 6.9928 & 1.3136 \\
\hline
\end{tabular}

The results also include angular trajectory and angular velocity of both pendulum arms and speed of computation. Figure 7 shows the angles obtained and the angular velocities. Since the readings are noisy so Savitzky Golay smoothing filter is applied. It smoothens the movement of the first arm. The algorithms miss out the movement of the second limb if the movement is really fast. This can be dealt with by increasing the camera resolution. Use of a better camera with a frame rate of nearly 72 fps (Ali, 2015) can improve the results significantly. If the lighting of the image acquisition platform is made better, the results can be further improved significantly.

\section{CONCLUSION}

In this paper we explored the efficacy of estimating the pose of a double pendulum using image processing thus reducing the cost of installing setups based on encoders, accelerometers and gyroscopes. Four methods were compared against a reference method using metrics such as accuracy, the speed of computation, and the memory usage. The results suggest that $4^{\text {th }}$ method has the highest computation speed and the lowest error for the first limb. This method's $\left(4^{\text {th }}\right)$ error is highly dependent on the width of the pendulum. Since this method searches for a white pixel in a certain specified region, the smaller is the width of the pendulum, the smaller is the deviation from the truth. However for the second limb the Canny edge detection followed by Hough transform gives the lowest error $\left(1^{\text {st }}\right.$ method). All the methods require a uniform background. The results highlight the speed-accuracy-memory tradeoff between the methods and could be used to identify the appropriate method for a given application based on the required performance metric.

\section{REFERENCES}

Ali D. and Teerapong D., 2015, Control of Double Inverted Pendulum First Approach, Blekinge Institute of Technology Artega M. A. and Siciliano B., 2000, On Tracking Control of Flexible Robot Arms, IEEE Transactions on Automatic Control, vol. 45

Ataei M. et al, 2007, The control of a double-pendulum system, International Conference on Control, Automation and Systems

Bergues G. J., 2019, Straight Line Detection Through Sub-pixel Hough Transform, Intelligent Computing, vol. 998

Canny J., 1986, A Computational Approach to Edge Detection, IEEE Transactions on Pattern Analysis and Machine Intelligence, vol. PAMI-8

Gonzalez C., 1977, Digital Image Processing, Upper Saddle River, NJ: Pearson/Prentice Hall

Kavithaa R. et al, 2013, Simple Pendulum analysis - A Vision based approach, Fourth International Conference on Computing, Communications and Networking Technologies

Kiril A., Iva N., 2013, An algorithm for error reducing in IMU, 2013 IEEE INISTA

Kulp C. W. et al, 2007, Tracking the Motion of a Double Pendulum Using Mathematica, Journal of Planning Education and Research, vol. 12

Lee J. D. and Wang B. L., 1998, Optimal control of a flexible robot arm, Computers and Structures, vol. 29

Miroslav H. and Peter K., 2018, About Edge Detection in Digital Images, Radio Engineering, vol. 27, no. 4

Nguyen T. T., 2009, Rectangular object tracking based on standard Hough transform, IEEE International Conference on Robotics and Biomimetics

Richard O. D. and Peter E. H., 1972, Use of the Hough Transformation To Detect Lines and Curves in Pictures, Communications of $A C M$, vol. 15 , no. 1

Robinson J., 2010, Double Pendulum, Georgia Institute of Technology 